\title{
Initial studies on the lytic effects of burkholdines upon mycotic agents
}

\begin{abstract}
Exposure of yeast and fungi to burkholdines, metabolites produced by an isolate of Burkholderia ambifaria, lead to $3-\log$ reductions (99.9\% killing) in colony counts of Saccharomyces cerevisiae in $30 \mathrm{~min}$. Burkholdine exposure also resulted in rapid (30min) lysis and release of the intracellular enzyme maltase of cells of either $S$. cerevisiae or Aspergillus niger. Viability of $S$. cerevisiae, as measured by the LIVE/DEAD ${ }^{\circledR}$ Yeast Viability test, was abolished within 30min of burkholdine exposure. Loss of colony-forming units, loss of viability, and release of maltase were highly correlated over time, suggesting that the basis for the anti-fungal activity of burkholdines is the loss of membrane integrity.
\end{abstract}

Keywords: burkholdines, burholderia ambifaria, yeast, fungi, killing, lysis
Volume 5 Issue 2 - 2017

\author{
Joseph O Falkinham \\ Department of Biological Sciences, Virginia Tech, USA
}

Correspondence: Joseph O Falkinham, Department of Biological Sciences, I 405 Perry Street, Blacksburg,VA 2406I0406, USA, Tel I54023 I593।, Fax I54023 I 9307 ,

Email jofiii@vt.edu

Received: July 12, 2017 | Published: July 19, 2017

\section{Introduction}

It has become clear that there is a need for novel anti-fungal agents whether for medicine or agriculture. For example, new anti-fungal agents are needed for the treatment of plant pathogenic fungi due to the emergence of azole-resistant isolates of plant pathogenic fungi. Burkholdines, produced by an isolate of Burkholderia ambifaria are novel anti-yeast and antifungal compounds. ${ }^{2}$ The burkholdines are lipopeptide antibiotics and strain $2.2 \mathrm{~N}$ produces as many as 8 related burkholdines of molecular weight 1097-1229.,3 Field trials showed activity against a wide variety of fungi that infect crops; for example Septoria tritici and Septoria nodorum in wheat, Phytophthora infestans in tomato, and Pyricularia oryzae in rice and tomato. ${ }^{5,6}$ In addition, burkholdines have strong anti-fungal activity against Mycosphaerella fijiensis, ${ }^{5,6}$ a pathogen of bananas that is responsible for considerable economic loss.?

In addition to displaying anti-fungal activity against plant pathogenic fungi, the burkholdines have strong activity against the medically important yeast Candida albicans and Cryptococcus neoformans and the filentoamus fungus, Aspergillus niger. ${ }^{2}$ Infections caused by yeast and fungi have a significant impact on human health in the United States. For example, the emergence of azole-resistant Candida albicans leads to significant increases in morbidity and mortality of those infection. ${ }^{8}$ Further, a population-based survey in the United States demonstrated that the incidence of hospital-acquired Candida spp. infections was 8 cases per $100,000^{9}$ and based on an estimate of the cost of treating each Candida spp. infected patient, the total cost of nosocomial Candida spp. infections in the United States would be $\$ 800$ million per year. ${ }^{9}$ In addition to azole-resistance in Candida spp., azole-resistance has emerged in Aspergillus fumigates..$^{10}$ Further, azole-resistance in A. funigatus has been linked with agricultural use of azole fungicides.

Microscopic observations of suspensions of $S$. cerevisiae exposed to burkholdines demonstrated significant short term $(<30 \mathrm{~min})$ lysis. That observations prompted an investigation to determine the mechanism of the basis for the anti-fungal activity of the burkholdines.

\section{Materials and methods}

\section{Burkholdines}

The source of burkholdines was a $1 \%(\mathrm{wt} / \mathrm{vol})$ suspension of spray dried cells of B. ambifaria strain $2.2 \mathrm{~N}$ in sterile tap water. One gram of the spray dried cells had an equivalent activity of $0.006 \mathrm{gm}$ of purified burkholdines $^{3,4}$ and had an MIC of $0.5 \mu \mathrm{g} / \mathrm{mL}$ against $S$. cerevisiae and C. albicans and $1.0 \mu \mathrm{g} / \mathrm{mL}$ against $A$. niger.

\section{Microbial strains and growth}

Saccharomyces cerevisiae, Candida albicans, and Aspergillus niger cultures ${ }^{2}$ were grown to mid log phase in $10 \mathrm{~mL}$ of Yeast Extract-Peptone-Maltose Broth (YEPM) for $6 \mathrm{hr}$ at $32^{\circ} \mathrm{C}$. Cultures were streaked on Yeast Extract Peptone Dextrose (YEPD) agar and incubated at $32^{\circ} \mathrm{C}$ for $24-48 \mathrm{hr}$. to confirm purity and colony morphology. Yeast or fungal mycelium was collected by centrifugation $(5,000 \mathrm{xg}$ for $20 \mathrm{~min})$ and after discarding the supernatant medium, cells or mycelium were washed in $10 \mathrm{~mL}$ of sterile tap water and finally suspended in an equal volume of sterile tap water.

\section{Measurement of killing, lysis, and membrane damage}

Killing of S. cerevisiae and C. albicans cells was measured as the reduction in colony-forming units (CFU) over time. In a $125 \mathrm{~mL}$ flask, $20 \mathrm{~mL}$ of a cell suspension was mixed with $2.0 \mathrm{~mL}$ of the burkholdine suspension and immediately and at 1,2 , and $3 \mathrm{hr}$ CFU were measured by spreading $0.1 \mathrm{~mL}$ (in triplicate) of the undiluted suspension and dilutions in sterile tap water on YEPD agar and incubating at $32^{\circ} \mathrm{C}$. Results were expressed as $\mathrm{CFU} / \mathrm{mL}$ and survival calculated as a percentage of the initial count. Lysis of $S$. cerevisiae and A. niger was measured as release of the intracellular enzyme maltase. ${ }^{11}$ Using the same suspension as used for measuring CFU reduction, $0.5 \mathrm{~mL}$ of the reaction was transferred to the filter housing of a Spin-X tube containing para-nitrophenyl maltose and centrifuged at $16,000 \mathrm{x}$ g for $2 \mathrm{~min}$. The filter housing with cells was removed and the filtrate in the Spin-X tube incubated at $37^{\circ} \mathrm{C}$ for $30 \mathrm{~min}$, the reaction stopped and the red color of para-nitrophenol intensified by adding $0.25 \mathrm{~mL}$ of 
$1 \mathrm{M} \mathrm{Na} 2 \mathrm{CO} 3$, and the absorbance at $450 \mathrm{~nm}$ measured. The positive control for maltase activity was a $0.45 \mu \mathrm{m}$ pore size-filtered sonicated suspension of $S$. cerevisiae or A. niger. Results are reported as the percent of maltase activity of the appropriate sonicated controls. Viability of $S$. cerevisiae cells was assessed using the LIVE/DEAD Yeast Viability Kit (Molecular Probes, Eugene, OR) as described by the manufacturer using the same suspension as used for measuring CFU reduction and lysis.

\section{Results}

\section{Killing, lysis, and viability of s. cerevisiae exposed to burkholdines}

In Table 1 are displayed the data on survival/killing, lysis, and viability of $S$. cerevisiae cells upon exposure to the burkholdines.
Yeast cells, grown, prepared, and exposed to burkholdines as described, were rapidly killed as measured by loss of colony-forming units. After $30 \mathrm{~min}$ exposure, $99.79 \%$ of the $S$. cerevisiae cells were unable to form colonies. Longer exposure, specifically $60 \mathrm{~min}$, led to 3-logs of killing (Table 1).

Burkholdine-exposure led to lysis of S. cerevisiae (Table 1). Lysis of the yeast cells was measured by the release of the intracellular enzyme maltase. ${ }^{8,9}$ The results, expressed as percentage of released maltase activity compared to a sonicate of $S$. cerevisiae cells show that lysis could be detected after 60min exposure (Table 1). Longer exposure, namely to $180 \mathrm{~min}$ led to release of almost $20 \%$ of the activity of the sonicated control (Table 1). The correlation between percentage killing and percentage of released maltase activity was high $(\mathrm{r} 2=0.8077)$.

Table I Burkholdine-mediated killing, lysis and loss of viability of S. cerevisiae

\begin{tabular}{lllll}
\hline Exposure & Survival $^{\mathrm{a}}$ & Percent killing $^{\mathrm{b}}$ & Percent lysis $^{\mathrm{c}}$ & Percent dead $^{\mathrm{d}}$ \\
\hline 0 & 100 & 0 & 0 & 43 \\
$30 \mathrm{~min}$ & 0.21 & 99.79 & 0 & 62 \\
$60 \mathrm{~min}$ & 0.088 & 99.912 & $3.95 \pm 0.21$ & 68 \\
$120 \mathrm{~min}$ & 0.065 & 99.935 & $11.6 \pm 0.14$ & 98 \\
$180 \mathrm{~min}$ & 0.058 & 99.942 & $19.85 \pm 1.48$ & 96 \\
\hline
\end{tabular}

aExpressed as surviving fraction of CFU of untreated control

bExpressed as percent of CFU reduction

'Expressed as percent of released maltase activity of sonicated control

dExpressed as percent of inviable (red) cells of untreated control

Using the LIVE/DEAD ${ }^{\circledR}$ Yeast Viability Kit (Molecular Probes, Eugene, OR) loss of viability as expressed as percent dead (red) $S$. cerevisiae cells was quite rapid (Table 1). The high percentage of dead cells in the 0 exposure control was likely due to the fact that the assay required $30 \mathrm{~min}$ incubation after addition of the dyes before epifluorescence microscopy and examination of cells. As noted for both the reduction in colony-forming units and the increase in maltase activity released from cells, as the duration of burkholdines increased, the percentage of dead cells increased (Table 1). In addition, the extent of killing as loss of CFU and viability as percent dead cells for $S$. cerevisiae were correlated $(\mathrm{r} 2=0.6394)$.

\section{Burkholdine-induced lysis and loss of viability of Aspergillus niger cells}

To confirm that the lytic activity of the burkholdines were not restricted to yeast, lysis and viability of $A$. niger hyphae were measured. Although it was not possible to reproducibly measure the killing of $A$. niger cells as colony-forming units, it was possible to measure the effect of burkholdine exposure on the release of intra cellular maltase and the loss of viability of $A$. niger cells. Washed hyphae of $A$. niger were exposed to burkholdines and hyphae-free filtrates assayed for maltase activity. Released maltase activity, expressed as a percentage of a sonicated hyphae control was $25 \%$. Measurement of the viability of burkholdine-exposed hyphae showed that the percentage of dead (red) A. niger hyphae was $100 \%$ after $30 \mathrm{~min}$ exposure.

\section{Discussion}

The source of burkholdines was a sonicated $1 \%(\mathrm{wt} / \mathrm{vol})$ suspension of spray dried cells of B. ambifaria strain $2.2 \mathrm{~N}$. The cells were spray dried at $170^{\circ} \mathrm{C}$ inlet temperature and contained no viable cells (i.e., $<10 \mathrm{CFU} / \mathrm{gm}$ ) and lacked all but anti-fungal and anti-yeast activity. Although a single purified burkholdine was not employed, to date all members of the burkholdine family produced by strain $2.2 \mathrm{~N}$ have equal specific activity. ${ }^{3,4}$ In the sonicated suspension used here, anti-yeast and anti-fungal activity would have been due to all related burkholdines; no synergistic activity has been demonstrated between individual burkholdines. ${ }^{3,4}$

Killing of yeast cells as measured by decrease of colony-forming units coincided with the release of intracellular maltase and loss of viability (Table 1). The short time needed to exhibit killing, lysis, and loss of viability suggests that they reflect the primary result of burkholdine activity, not a secondary consequence of some other effect (e.g., ATP reduction). Based on the observation that burkholdine exposure results in rapid lysis of both $S$. cerevisiae and A. niger cells suggests that the primary target is a common element of the cell membranes of both.

\section{Acknowledgements}

None.

\section{Conflict of interest}

The author declares no conflict of interest.

\section{References}

1. Chowdhary A, Kathuria S, Xu J, et al. Emergence of azole-resistant Aspergillus fumigatus strains due to agricultural azole use creates an increasing threat to human health. PLoS Pathogens. 2013;9(10):e1003633. 
2. Cain CC, Henry AT, Waldo RH, et al. Identification and characteristics of a novel Burkholderia strain with broad-spectrum antimicrobial activity. Appl Environ Microbiol. 2000;66(9):4139-4141.

3. Tawfik KA, Jeffs P, Bray B, et al. Burkholdines 1097 and 1229, potent antifungal peptides form Burkholderia ambifaria 2.2 N. Organic Letters. 2009;12(4):664-666.

4. Lin Z, Falkinham JO, Tawfik KA, et al. Burkholdines from Burkholderia ambifaria: antifungal agents and possible virulence factors. J Nat Prod. 2012;75(9):1518-1523.

5. Falkinham JO, Cassida LE, Cain CC. Non-Obligate predatory bacterium Burkholderia casidae. US Patent Number 6,319,497. 2001.

6. Falkinham JO, Cassida LE, Cain CC. Non-Obligate predatory bacterium Burkholderia casidae. US Patent Number 6,689,357. 2004.
7. Marin DH, Romero RA, Guzmán M, et al. Black sigatoka: an increasing threat to banana cultivation. Plant Dis. 2003;87(3):208-222.

8. Tobudic S, Kratze C, Prester E. Azole-resistant Candida spp - - emerging pathogens? Mycoses. 2012;55(Suppl 1):24-32.

9. Miller LG, Hajjeh RA, Edwards JE. Estimating the cost of nosocomial candidemia in the United States. Clin Infect Dis. 2001;32(7):1110.

10. Pham CD, Reiss E, Hagen F, et al. Passive surveillance for azoleresistant Aspergillus fumigates, United States, 2011-2013. Emerg Infect Dis. 2014;20(9):1498-1503.

11. Jewell SN, Waldo RH, Cain CC, et al. Rapid detection of lytic antimicrobial activity against yeast and filamentous fungi. $J$ Microbiol Methods. 2002;49(1):1-9. 